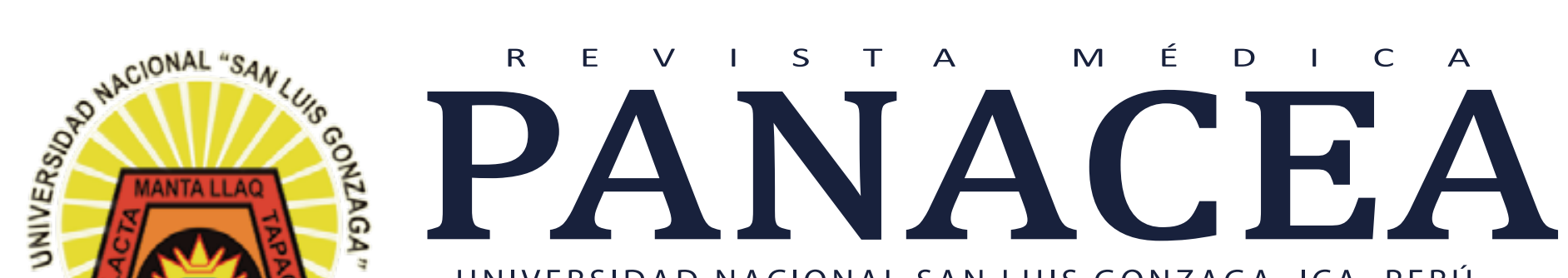

UNIVERSIDAD NACIONAL SAN LUIS GONZAGA. ICA, PERÚ

FACULTAD DE MEDICINA HUMANA "DANIEL ALCIDES CARRIÓN"

p-ISSN 2223-2893

e-ISSN 2225-6989

Volumen 9 Número 2 PUBLICACION CUATRIMESTRAL Mayo - Agosto

2020

DOI: https://doi.org/10.35563/rmp.v9i2.324

ARTÍCULO ORIGINAL:

ANÁLISIS PSICOMÉTRICO DEL INSTRUMENTO DE CLIMA ORGANIZACIONAL PARA EL PERSONAL DEL MINISTERIO DE SALUD DEL PERÚ.

PSYCHOMETRIC ANALYSIS OF THE ORGANIZATIONAL CLIMATE INSTRUMENT FOR MINISTRY OF HEALTH PERSONNEL IN PERU.

\title{
AUTORES:
}

Bladimir Becerra Canales

Consuelo Solari Bonifacio

Domizbeth Becerra Huaman

INDEXADA EN:

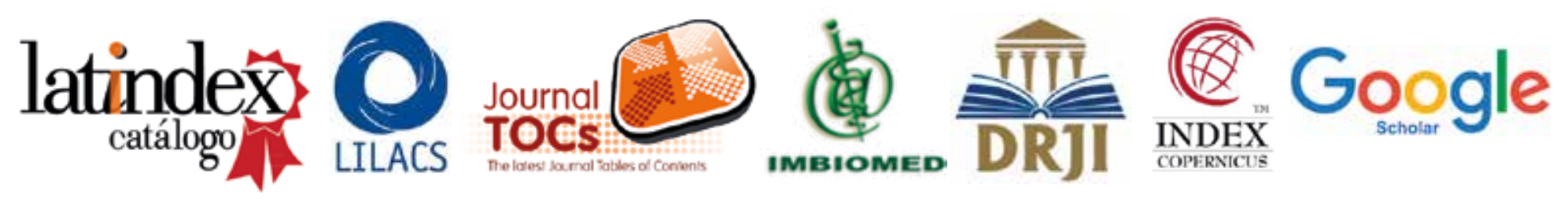

revistas.unica.edu.pe 


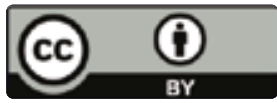

\section{ANÁLISIS PSICOMÉTRICO DEL INSTRUMENTO DE CLIMA ORGANIZACIONAL PARA EL PERSONAL DEL MINISTERIO DE SALUD DEL PERÚ.}

PSYCHOMETRIC ANALYSIS OF THE ORGANIZATIONAL CLIMATE
INSTRUMENT FOR MINISTRY OF HEALTH PERSONNEL IN PERU.

Bladimir Becerra-Canales ${ }^{1,2, a}$, Consuelo Solari-Bonifacio ${ }^{3, b}$, Domizbeth Becerra-Huaman ${ }^{4, c, d}$

\author{
1. Dirección Regional de Salud. Ica, Perú. \\ 2. Universidad Autónoma de Ica. Ica, Perú. https://orcid.or- \\ g/0000-0002-2234-2189 \\ 3. Universidad Privada San Juan Bautista. Chincha, Perú \\ 4. Universidad San Luis Gonzaga de Ica, Perú. \\ a. Cirujano Dentista. Doctor en Salud Pública.
}

b. Medico Maestro en Medicina Humana, Especialista en Administración en Salud.

c. Sociedad Científica de Estudiantes de Medicina de Ica (SOCEMI). Perú.

d. Estudiante de Medicina Humana

DOI: https://doi.org/10.35563/rmp.v9i2.324

\section{Correspondencia:}

Bladimir Becerra Canales

Dirección: CC. HH La Angostura III Etapa $\mathrm{H}-10$,

Subtanjalla. Ica, Perú.

Teléfono: (+51) 956690060

Correo electrónico:

icapredica@gmail.com

Contribuciones de autoría: BBC: concepción y diseño del estudio, análisis estadístico, discusión y revisión final de manuscrito. CSB: recolección de los datos y revisión crítica del manuscrito. $\mathrm{DBH}$ : recolección e interpretación de los datos y discusión.

Conflicto de intereses: no existen conflictos de intereses del autor o autores de orden económico, institucional, laboral o personal.

Financiamiento: Autofinanciado.

\section{Cómo citar:}

Becerra-Canales

Solari-Bonifacio organizacional para el personal del ministerio de salud del Perú. Rev méd panacea.2020;9(2): 74-81. DOI:

https://doi.org/10.35563/r mp.v9i2.324

Recibido: 24 - 06 - 2020 Aceptado: 08 - 07 - 2020 Publicado: 20 - 08 - 2020

\section{RESUMEN}

Objetivo: Evaluar las propiedades psicométricas del instrumento que mide el clima organizacional en el personal de las Instituciones Prestadoras de Servicios de Salud del Ministerio de Salud del Perú. Materiales y métodos: Estudio de tipo instrumental, participaron 104 trabajadores de la Dirección Regional de Salud Ica, seleccionados por muestreo no probabilístico por disposición. Realizamos análisis de la variabilidad y correlación de los ítems, un análisis factorial exploratorio (AFE) para valorar la estructura interna de la escala y análisis factorial confirmatorio (AFC) que incluyó el método de estimación de máxima verosimilitud robusta; finalmente evaluamos la confiabilidad del instrumento con el Alpha de Cronbach. Resultados: Los hallazgos confirman índices de variabilidad de los ítems de 0,41 a 0,89; con valores de correlación ítem-total de 0,28 hasta 0,64; el análisis factorial confirmatorio mostró una estructura de tres dimensiones para la sub-variable cultura de la organización que explicaba el 71,58\% de la varianza total; cuatro dimensiones para diseño organizacional que explicaba $70,41 \%$ de la varianza total y cuatro dimensiones para la sub-variable potencial humano, que explicaba $67,42 \%$ de la varianza total. Los índices de bondad de ajuste no fueron los ideales en los modelos analizados. La consistencia interna de la escala global mostró un índice aceptable $(\alpha=0,88)$ y las sub-variables cultura de la organización $(\alpha=0,75)$; diseño organizacional $(a=0,67)$ y potencial humano $(a=0,81)$. Conclusiones: El instrumento presenta un modelo factorial indefinido en su estructura interna y confiablidad adecuada; por lo tanto, puede ser usado en futuras investigaciones y estudios de validación.

Palabras clave: Clima organizacional; Ministerio de salud; Psicometría (fuente: DeCS BIREME).

\section{ABSTRACT}

Objective: To evaluate the psychometric properties of the instrument that measures the organizational climate in the personnel of public health centers of the Ministry of Health of Peru. Materials and methods: Instrumental type study, 104 workers from the Regional Health Directorate of Ica participated, selected by non-probabilistic sampling by disposition. We performed variability and correlation analysis of the items, an exploratory factorial analysis (AFE) to assess the internal structure of the scale and confirmatory factorial analysis (AFC) that included the method of maximum robust likelihood estimation; finally we evaluated the reliability of the instrument with Cronbach's Alpha. Results: The findings confirm item variability indices from 0.41 to 0.89 ; with item-total correlation values from 0.28 to 0.64 ; confirmatory factor analysis showed a three-dimensional structure for the sub-variable organizational culture explaining $71.58 \%$ of the total variance; four dimensions for organizational design explaining $70.41 \%$ of the total variance and four dimensions for the sub-variable human potential, explaining $67.42 \%$ of the total variance. Goodness-of-fit indices were not ideal in the models analyzed. The internal consistency of the global scale showed an acceptable index $(a=0.88)$ and the sub-variables organizational culture $(a=0.75)$; organizational design ( $a$ $=0.67$ ) and human potential $(a=0.81)$. Conclusions: The instrument presents an undefined factorial model in its internal structure, however it can be used in future research and validation studies.

Keywords: Organizational climate; Ministry of health; Psychometry. (Source: MeSH NLM). 


\section{INTRODUCCIÓN}

El clima organizacional es una variable subjetiva un constructo complejo de varias dimensiones (1) que tiene la finalidad de caracterizar y/o medir una realidad organizacional (2) definiendo escenarios (3) multidimensionales y complejos $(4,5)$. Se le relaciona frecuentemente con la cultura organizacional, sin embargo el clima es temporal y la cultura es más arraigado (6).

La conceptualización del término hace referencia a un conjunto de propiedades del ambiente de trabajo que directa o indirectamente inciden sobre las conductas de los trabajadores motivándolos o desmotivándolos $(7,8)$ y que pueden ser medida por medio de instrumentos documentales basadas en las percepciones que el individuo tiene acerca de su ambiente de trabajo (9). Estas percepciones son cuantificadas por los ítems y dimensiones de un instrumento documental que mide el clima laboral (10), y definen un patrón de interacciones de las personas en la organización sanitaria (3) e identifican elementos intervinientes que contextualizan la realidad organizacional (11).

El estudio del clima organizacional, permite conocer, en forma científica y sistemática, las opiniones de las personas acerca de su entorno laboral y condiciones de trabajo, con el fin de elaborar planes que permitan superar de manera priorizada los factores negativos que se detecten y que afectan el compromiso y la productividad del potencial humano (12). La elaboración del estudio del clima organizacional es un proceso sumamente complejo a raíz de la dinámica de la organización, del entorno y de los factores humanos. Por tanto muchas organizaciones reconocen que uno de sus activos fundamentales es su factor humano y requieren contar con mecanismos de medición periódica de su Clima Organizacional (12).

Los instrumentos de clima organizacional, son reconocidos como herramienta gerencial para la toma de decisiones (13). La revisión de la literatura reportan diversos instrumentos para estudiar o medir el clima organizacional (14,15). En consecuencia cada vez estos se constituyen como una valiosa herramienta diagnóstica para la gestión del cambio en busca del logro de una mayor eficiencia organizacional, condición indispensable en el mundo actual $(16,11)$, caracterizado por la globalización, la competitividad, la productividad, el manejo de la información y el permanente desarrollo corporativo.

Si bien es cierto, se han desarrollado varios instrumentos dirigidos a medir el clima organizacional, sin embargo la mayoría de estos fueron desarrollados en otros idiomas y en poblaciones diferentes. De ahí, que urge la necesidad de contar con instrumentos contextualizados y de adecuadas propiedades métricas. Por tal razón, el estudio tuvo como objetivo evaluar las propiedades psicométricas del instrumento que mide el clima organizacional en el personal de las Instituciones Prestadoras de Servicios de Salud del Ministerio de Salud del Perú.

\section{MATERIALES Y MÉTODOS}

Diseño de estudio, población y muestra: Se realizó un estudio transversal, analítico, de tipo instrumental. La población de estudio fueron trabajadores de la Dirección
Regional de Salud Ica, de ambos sexos que tengan 18 años o más. Se excluyó a aquellos que no aceptaron participar en el estudio o tuvieron algún tipo de discapacidad que les impida responder el cuestionario. El tamaño de la muestra fue de 104 trabajadores, seleccionados por disponibilidad de tipo no probabilístico.

Descripción del instrumento: El instrumento ha sido construido por el Comité Técnico de Clima Organizacional del Ministerio de Salud, con la participación de un equipo de expertos intersectorial. Ha sido validado por juicio de expertos, así mismo a través de la metodología de focus groups se realizó la validez de la claridad de los enunciados (12).

El instrumento consta de: i) Datos generales: Se consideró la Edad, sexo, grupo ocupacional y condición laboral; ii) Enunciados: Se presentan 34 enunciados, 6 de los cuales corresponden a la escala de sinceridad (Ítems 2, 9, 15, 22, 28 y 32) y 28 reactivos de opción de respuesta múltiple (nunca, a veces, frecuentemente y siempre), que miden las tres sub variables y 11 dimensiones del clima organizacional:

Tabla 1: Variables, dimensiones y reactivos que componen el instrumento de medición del clima organizacional del MINSA.

\begin{tabular}{|c|c|c|}
\hline Sub variables & Dimensiones & Ítems \\
\hline \multirow[t]{3}{*}{ Cultura de la Organización } & Motivación & $1,8,33$ \\
\hline & Identidad & $20,23,31$ \\
\hline & Conflicto y Cooperación & 24,26 \\
\hline \multirow[t]{4}{*}{ Diseño Organizacional } & Toma de Decisiones: & 3,14 \\
\hline & Remuneración & $\quad 6,27$ \\
\hline & Estructura & $\quad 10,13$ \\
\hline & Comunicación Organizacional & $29,30,34$ \\
\hline \multirow[t]{4}{*}{ Potencial Humano } & Innovación & $4,5,12,17$ \\
\hline & Liderazgo & 7,19 \\
\hline & Recompensa & $11,16,21$ \\
\hline & Confort & 18,25 \\
\hline
\end{tabular}

Procedimientos: Entre noviembre y diciembre del 2019, se coordinó fechas y horas para la recogida de información. Una vez que se confirmó que el participante cumplía con los criterios de inclusión, se le explicó el propósito del estudio y sus procedimientos, se obtuvo el consentimiento verbal y se procedió a realizar la encuesta heteroadministrada buscando privacidad en los casos donde encontramos más de un potencial participante.

Se realizó un control de calidad de la información recolectada en el trabajo de campo, verificando el contenido del instrumento y codificando adecuadamente las variables del estudio.

Análisis estadístico: Se describieron las características generales de la población objeto de estudio mediante frecuencias absolutas y relativas para las variables categóricas, así como medidas de tendencia central y dispersión para las variables numéricas.

Se incluyó un análisis de la variabilidad de los ítems, considerándose como aceptables los valores superiores a 0,20; dado que, al tratarse de una escala de tipo Likert, se acepta un valor mínimo de 0,20 para poder afirmar que los ítems discriminan (18); así mismo un análisis de la matriz de 
correlación de los ítems e ítem total, mediante el coeficiente de correlación de Pearson (considerándose como apropiados los valores superiores a 0,50). Previo al análisis factorial, se determinó la existencia de relaciones significativas entre las variables, con el Test de Esfericidad de Bartlett y el test de Kaiser-Meyer-Olkin (KMO), tomando como un correcto ajuste de la muestra promedio y por ítem cuando los valores fueron superiores a 0,7 (19).

Se realizó un análisis de fiabilidad para escalas y la consistencia interna fue medida con el Alpha de Cronbach; garantizando que estos índices superen el mínimo recomendado por la literatura $(, 70)$ para ser considerado un instrumento confiable (20).

Se consideró un valor de $\mathrm{p}<0,05$ como estadísticamente significativo. Para el procesamiento de los datos se usó el paquete estadístico "Statistical Package for the Social Sciences" para Windows versión 25.0 en Español.

Consideraciones éticas: Se informó a los participantes sobre el propósito de la investigación, el carácter voluntario y anónimo de su colaboración. Se destacó que la participación podía interrumpirse en el momento que el participante lo deseara. Se obtuvo el consentimiento verbal y no se ofreció ningún tipo de contraprestación.

\section{RESULTADOS}

Se encuestaron a 104 trabajadores, la mayoría de los cuales fueron de sexo femenino (66,3\%); grupo ocupacional administrativo (74,0\%); condición nombrado (79,8\%); el

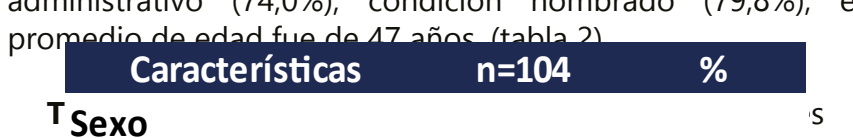

$\begin{array}{lll}\text { Masculino } & 35 & 33,7 \\ \text { Femenino } & 69 & 66,3\end{array}$

\section{Grupo ocupacional}

$\begin{array}{lll}\text { Administrativo } & 77 & 74 \\ \text { Asistencial } & 27 & 26\end{array}$

\section{Condición}

\begin{tabular}{ccc} 
Nombrado & 83 & 79,8 \\
Contratado & 21 & 20 \\
Edad, Media (DE) & $47,8(12,03)$ & \\
\hline n= muestra; F= Frecuencia absoluta; \\
$\%=$ Frecuencia relativa; $D E=$ Desviación
\end{tabular}

El instrumento en su versión original, reveló que la capacidad discriminante de los reactivos, es en general aceptable encontrando índices de variabilidad superiores a 0,41 ; con una media de 0,60; se constata que todos los ítems son homogéneos en términos de varianza, siendo adecuados para discriminar entre sujetos con valores distintos, en la variable medida. El análisis de la matriz de correlación (Coeficiente de Pearson), reveló correlaciones positivas entre los ítems, con valores de correlación ítems-total, desde 0,30 a 0,77 ( $p<0,00)$, estos valores significan que la magnitud de correlación es baja a moderada respectivamente. El ítem 10, es el único reactivo con valor de correlación menor. (tabla 3).

Tabla 3. Análisis de la variabilidad de los ítems y matriz de correlación.

\begin{tabular}{|c|c|c|c|c|}
\hline Sub variables & Dimensiones & Ítems & Varianza & $\begin{array}{c}\text { Correlación } \\
\text { (Suma) }\end{array}$ \\
\hline Cultura de la & Motivación & 1 & 0,594 & 0,603 \\
\hline \multirow[t]{8}{*}{ Organización } & & 8 & 0,676 & 0,412 \\
\hline & & 33 & 0,604 & 0,642 \\
\hline & Identidad & 20 & 0,686 & 0,773 \\
\hline & & 23 & 0,459 & 0,583 \\
\hline & & 31 & 0,466 & 0,519 \\
\hline & Conflicto y Cooperación & 24 & 0,697 & 0,613 \\
\hline & & 26 & 0,538 & 0,684 \\
\hline & & Total & 13,721 & \\
\hline \multirow[t]{10}{*}{ Diseño Organizacional } & Toma de Decisiones: & 3 & 0,758 & 0,681 \\
\hline & & 14 & 0,754 & 0,562 \\
\hline & Remuneración & 6 & 0,686 & 0,345 \\
\hline & & 27 & 0,783 & 0,457 \\
\hline & Estructura & 10 & 0,413 & 0,28 \\
\hline & & 13 & 0,703 & 0,508 \\
\hline & Comunicación & 29 & 0,617 & 0,673 \\
\hline & Organizacional & 30 & 0,894 & 0,641 \\
\hline & & 34 & 0,468 & 0,382 \\
\hline & & Total & 15,712 & \\
\hline \multirow[t]{12}{*}{ Potencial Humano } & Innovación & 4 & 0,586 & 0,71 \\
\hline & & 5 & 0,558 & 0,472 \\
\hline & & 12 & 0,493 & 0,665 \\
\hline & & 17 & 0,494 & 0,639 \\
\hline & Liderazgo & 7 & 0,648 & 0,582 \\
\hline & & 19 & 0,685 & 0,648 \\
\hline & Recompensa & 11 & 0,55 & 0,643 \\
\hline & & 16 & 0,604 & 0,718 \\
\hline & & 21 & 0,532 & 0,333 \\
\hline & Confort & 18 & 0,47 & 0,484 \\
\hline & & 25 & 0,581 & 0,624 \\
\hline & & Total & 24,034 & \\
\hline
\end{tabular}

El Análisis Factorial Exploratorio y el test de esfericidad de Bartlett, para la variable cultura de la organización arrojaron un estadístico de 184,710; con un $\mathrm{p}=0,000$; la medida de adecuación muestral $\mathrm{KMO}$ fue de ,693 $(<0,70)$. La variable diseño organizacional mostró un estadístico de 141,878; con un $\mathrm{p}=0,000$; la medida de adecuación muestral KMO fue de ,589 $(<0,70)$ y la variable potencial humano un estadístico de 228,295; con un $\mathrm{p}=0,000$; la medida de adecuación muestral $\mathrm{KMO}$ fue de ,811. Dado que, el KMO resultó bajo en los dos primeros casos no se recomienda su factorización ya que nos permiten afirmar que las correlaciones pueden no ser significativas entre las variables. Sin embargo el AFC, sugirió 3 dimensiones para cada una de las variables de clima organizacional; reveló además que la ubicación de las dimensiones e ítems por su carga factorial es distinto al propuesto en el instrumento original (tabla 4). El comportamiento de los datos es similar cuando se analiza todas las variables en su conjunto (tabla 5) 
Análisis psicométrico del instrumento de clima organizacional para elpersonal del ministerio de salud del Perú.

Tabla 4. Matriz de componente rotado para cada una de las variables del clima organizacional

\begin{tabular}{|c|c|c|c|c|c|c|c|c|c|c|c|c|c|}
\hline \multicolumn{4}{|c|}{ Cultura de la organización } & \multicolumn{5}{|c|}{ Diseño organizacional } & \multicolumn{5}{|c|}{ Potencial humano } \\
\hline \multirow{2}{*}{ Ítems } & \multicolumn{3}{|c|}{ Componente } & \multirow{2}{*}{ Ítems } & \multicolumn{4}{|c|}{ Componente } & \multirow{2}{*}{ Ítems } & \multicolumn{4}{|c|}{ Componente } \\
\hline & 1 & 2 & 3 & & 1 & 2 & 3 & 4 & & 1 & 2 & 3 & 4 \\
\hline P23 & 0,899 & 0,143 & $-0,033$ & P3 & 0,857 & 0,052 & 0,065 & 0,086 & P5 & 0,765 & 0,101 & 0,006 & $-0,339$ \\
\hline P31 & 0,889 & 0,043 & $-0,034$ & P29 & 0,848 & 0,009 & 0,135 & 0,08 & P11 & 0,68 & 0,164 & 0,017 & 0,461 \\
\hline P20 & 0,651 & 0,431 & 0,266 & P30 & 0,642 & 0,324 & $-0,001$ & 0,081 & P4 & 0,655 & 0,444 & 0,056 & 0,019 \\
\hline P24 & 0,118 & 0,87 & $-0,097$ & P27 & 0,036 & 0,916 & 0,07 & 0,123 & P12 & 0,618 & 0,225 & 0,421 & $-0,025$ \\
\hline P26 & 0,108 & 0,704 & 0,337 & P6 & 0,204 & 0,872 & $-0,092$ & $-0,099$ & P7 & 0,216 & 0,776 & $-0,18$ & 0,13 \\
\hline P33 & 0,139 & 0,701 & 0,191 & P13 & 0,255 & 0,046 & 0,786 & $-0,047$ & P17 & 0,273 & 0,709 & 0,27 & $-0,186$ \\
\hline P8 & $-0,168$ & 0,1 & 0,85 & P10 & $-0,102$ & $-0,058$ & 0,755 & 0,093 & P25 & 0,06 & 0,706 & 0,277 & 0,195 \\
\hline \multirow[t]{4}{*}{ P1 } & 0,21 & 0,167 & 0,801 & P34 & 0,076 & 0,022 & $-0,021$ & 0,936 & P16 & 0,441 & 0,459 & 0,214 & 0,341 \\
\hline & & & & P14 & 0,226 & 0,017 & 0,436 & 0,486 & P18 & 0,016 & 0,119 & 0,899 & 0,109 \\
\hline & & & & & & & & & P19 & 0,425 & 0,085 & 0,545 & 0,363 \\
\hline & & & & & & & & & P21 & $-0,098$ & 0,09 & 0,161 & 0,839 \\
\hline
\end{tabular}

Método de extracción: análisis de componentes principales.

Método de rotación: Varimax con normalización Kaiser.

Tabla 5. Matriz de componente rotado para las 11 dimensiones e ítems en su conjunto del clima organizacional.

\begin{tabular}{|c|c|c|c|c|c|c|c|c|c|c|c|}
\hline \multirow{2}{*}{ Ítems } & \multicolumn{11}{|c|}{$\begin{array}{c}\text { Clima organizacional } \\
\text { Componente }\end{array}$} \\
\hline & 1 & 2 & 3 & 4 & 5 & 6 & 7 & 8 & 9 & 10 & 11 \\
\hline P7 & 0,879 & 0,133 & 0,037 & 0,088 & 0,065 & 0,095 & 0,136 & 0,099 & $-0,056$ & 0,011 & 0,035 \\
\hline P8 & 0,842 & 0,06 & 0,057 & 0,209 & $-0,083$ & 0 & 0,111 & $-0,026$ & 0,224 & $-0,071$ & 0,155 \\
\hline P29 & 0,819 & $-0,007$ & 0,185 & 0,173 & $-0,066$ & 0,127 & 0,055 & 0,054 & 0,157 & $-0,022$ & 0,104 \\
\hline P3 & 0,774 & 0,069 & 0,074 & 0,078 & 0,035 & 0,11 & 0,115 & $-0,04$ & $-0,114$ & 0,32 & $-0,036$ \\
\hline P1 & 0,392 & 0,16 & 0,149 & 0,208 & $-0,07$ & 0,33 & 0,229 & 0,345 & 0,351 & 0,088 & $-0,25$ \\
\hline P27 & 0,105 & 0,847 & 0,038 & 0,122 & 0,007 & 0,054 & $-0,169$ & $-0,069$ & 0,011 & 0,027 & 0,133 \\
\hline P6 & 0,073 & 0,834 & 0,18 & 0,087 & 0,017 & $-0,053$ & 0,22 & $-0,017$ & $-0,068$ & $-0,087$ & 0,026 \\
\hline P21 & 0,07 & 0,56 & 0,048 & $-0,093$ & $-0,072$ & 0,166 & $-0,29$ & $-0,081$ & 0,196 & 0,546 & $-0,025$ \\
\hline P18 & 0,081 & 0,067 & 0,888 & 0,111 & $-0,131$ & 0,092 & $-0,021$ & $-0,045$ & 0,054 & 0,013 & $-0,07$ \\
\hline P19 & 0,146 & 0,325 & 0,556 & 0,164 & 0,022 & 0,181 & 0,302 & $-0,242$ & 0,284 & 0,007 & 0,263 \\
\hline P16 & 0,276 & 0,231 & 0,54 & 0,326 & $-0,099$ & 0,103 & 0,094 & 0,088 & $-0,078$ & 0,102 & 0,214 \\
\hline P26 & 0,241 & 0,04 & 0,064 & 0,778 & 0,108 & 0,095 & 0,26 & 0,046 & 0,06 & $-0,022$ & 0,155 \\
\hline P25 & 0,382 & 0,038 & 0,284 & 0,663 & 0,198 & 0,203 & $-0,083$ & $-0,018$ & 0,044 & 0,234 & $-0,089$ \\
\hline P33 & 0,13 & 0,363 & 0,355 & 0,644 & 0,188 & 0,193 & $-0,004$ & $-0,105$ & $-0,067$ & 0,109 & $-0,143$ \\
\hline P31 & $-0,024$ & 0,042 & $-0,003$ & 0,181 & 0,879 & 0,06 & $-0,026$ & 0,167 & $-0,038$ & $-0,014$ & 0,042 \\
\hline P23 & $-0,08$ & $-0,018$ & $-0,236$ & 0,053 & 0,82 & 0,162 & 0,111 & 0,074 & 0,064 & 0,236 & 0,028 \\
\hline P24 & 0,088 & 0,098 & $-0,045$ & 0,409 & $-0,008$ & 0,73 & 0,131 & 0,04 & $-0,147$ & 0,168 & 0,231 \\
\hline P20 & 0,178 & $-0,02$ & 0,155 & 0,012 & 0,311 & 0,719 & 0,14 & 0,149 & 0,086 & 0,133 & $-0,109$ \\
\hline P34 & 0,198 & $-0,046$ & 0,225 & 0,027 & 0,504 & 0,553 & $-0,141$ & $-0,219$ & 0,125 & $-0,239$ & 0,004 \\
\hline P11 & 0,089 & 0,136 & 0,252 & 0,268 & 0,015 & 0,533 & 0,224 & 0,436 & 0,063 & 0,133 & 0,27 \\
\hline P5 & 0,18 & $-0,135$ & $-0,045$ & 0,031 & 0,009 & 0,111 & 0,839 & $-0,103$ & 0,026 & 0,014 & 0,123 \\
\hline P4 & 0,283 & 0,315 & 0,244 & 0,286 & $-0,004$ & 0,205 & 0,615 & $-0,079$ & $-0,073$ & $-0,098$ & $-0,268$ \\
\hline P12 & 0,135 & 0,01 & 0,443 & 0,141 & 0,086 & 0,038 & 0,51 & 0,384 & $-0,188$ & 0,273 & 0,064 \\
\hline P10 & 0,046 & $-0,165$ & $-0,114$ & $-0,068$ & 0,212 & 0,096 & $-0,15$ & 0,807 & 0,134 & 0,003 & 0,004 \\
\hline P13 & 0,228 & 0,033 & 0,122 & 0,071 & 0,131 & 0,019 & 0,008 & 0,159 & 0,748 & 0,274 & 0 \\
\hline P17 & 0,307 & 0,194 & 0,329 & 0,244 & 0,22 & 0,043 & 0,35 & 0,039 & $-0,536$ & 0,119 & 0,056 \\
\hline P14 & 0,137 & $-0,104$ & 0,089 & 0,236 & 0,227 & 0,147 & 0,123 & 0,093 & 0,223 & 0,647 & 0,124 \\
\hline P30 & 0,456 & 0,297 & 0,103 & 0,061 & 0,119 & 0,155 & 0,1 & 0,038 & $-0,047$ & 0,124 & 0,673 \\
\hline
\end{tabular}

Método de extracción: análisis de componentes principales.

Método de rotación: Varimax con normalización Kaiser.

Analizamos los índices de bondad de ajuste (tabla 6), y encontramos valores de ajuste aceptable para el modelo factorial de la variable diseño organizacional $(p=174 ; C F I=0,946 ; T L I=0,915)$; sin embargo las medidas de ajuste para el modelo factorial de la variable cultura de la organización ( $p=000 ; C F I=0,802 ; T L I=0,673)$ y potencial humano ( $p=0,053 ; C F I=0,918 ; T L I=0,887)$ no fueron los ideales. En consecuencia el análisis de las correlaciones entre los items y los factores sugieren que el modelo factorial propuesto en las dos variables del clima organizacional podría ser rechazado; dado que las medidas de la calidad de ajuste de los modelos factoriales propuestos no se ajustan de manera satisfactorias a los datos.

Tabla 6. Índices de bondad de ajuste de los modelos (variables) del instrumento.

\begin{tabular}{lccccccccc} 
& \multicolumn{3}{c}{$\begin{array}{c}\text { Medida de ajuste } \\
\text { absoluto }\end{array}$} & \multicolumn{3}{c}{ Medidas de ajuste incremental } & \multicolumn{3}{c}{ Medidas de ajuste de la parsimonia } \\
Variables & p-valor & RMSEA & CFI & TLI & NFI & PRATIO & PCFI & PNFI & AC \\
CO & 0 & 0,162 & 0,802 & 0,673 & 0,743 & 0,607 & 0,487 & 0,415 & 87,661 \\
DO & 0,174 & 0,06 & 0,946 & 0,915 & 0,805 & 0,639 & 0,604 & 0,514 & 73,185 \\
PH & 0,053 & 0,072 & 0,918 & 0,887 & 0,772 & 0,727 & 0,668 & 0,562 & 107,399 \\
\hline
\end{tabular}

$\mathrm{CO}=$ Cultura de la organización; $\mathrm{DO}=$ Diseño organizacional; $\mathrm{PH}=$ Potencial humano

RMSEA = raíz cuadrada media del error de aproximación; $\mathrm{CFI}=$ índice de ajuste comparativo; $\mathrm{TLI}$ = índice de Tucker-Lewis; $\mathrm{NFI}=$ indice de ajuste normado; $\mathrm{PRATIO}=$ razón de parsimoniosidad; $\mathrm{PCFI}$ = índice de bondad de ajuste de parsimonia; $\mathrm{PNFI}$ = índice de ajuste normado de la parsimonia; $\mathrm{AIC}=$ criterio de información de Akaike. 
No obstante las cargas factoriales fueron mayores de 0,50; lo que daría soporte a un modelo de tres dimensiones de la variable cultura de la organización (figura 1), de cuatro dimensiones de la variable diseño organizacional (figura 2) y de cuatro dimensiones de la variable potencial humano (figura 3).

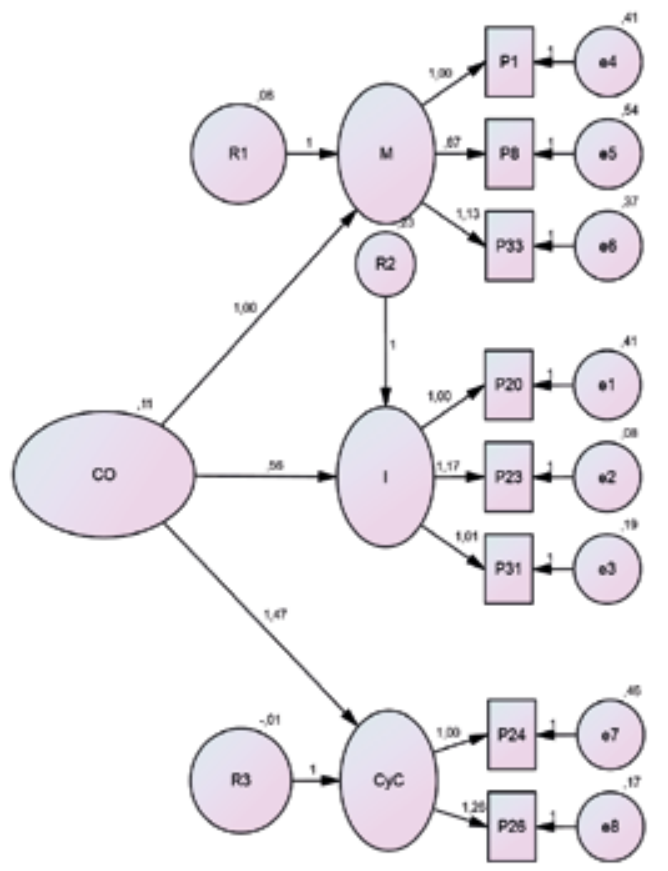

Figura 1. Estructura interna del modelo de tres dimensiones de la variable cultura de la organización.

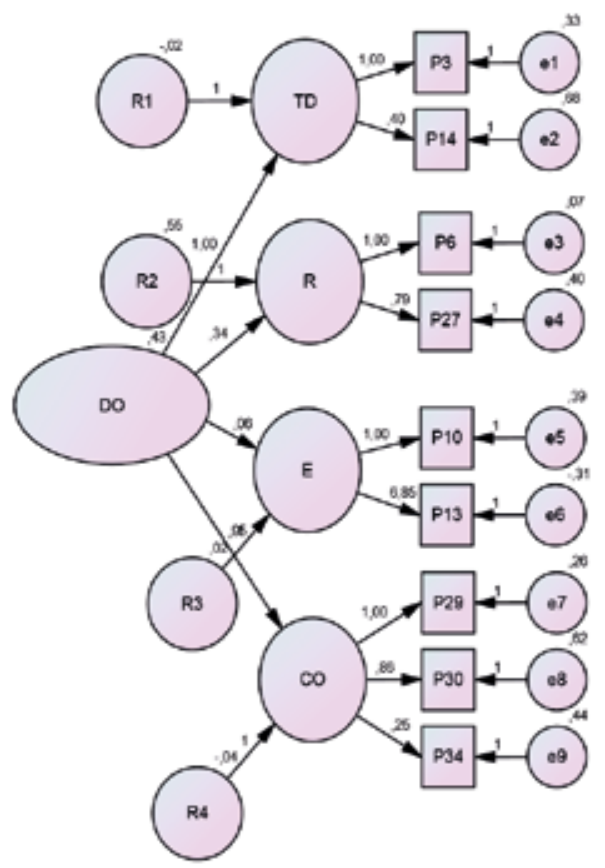

Figura 2. Estructura interna del modelo de cuatro dimensiones de la variable diseño organizacional.

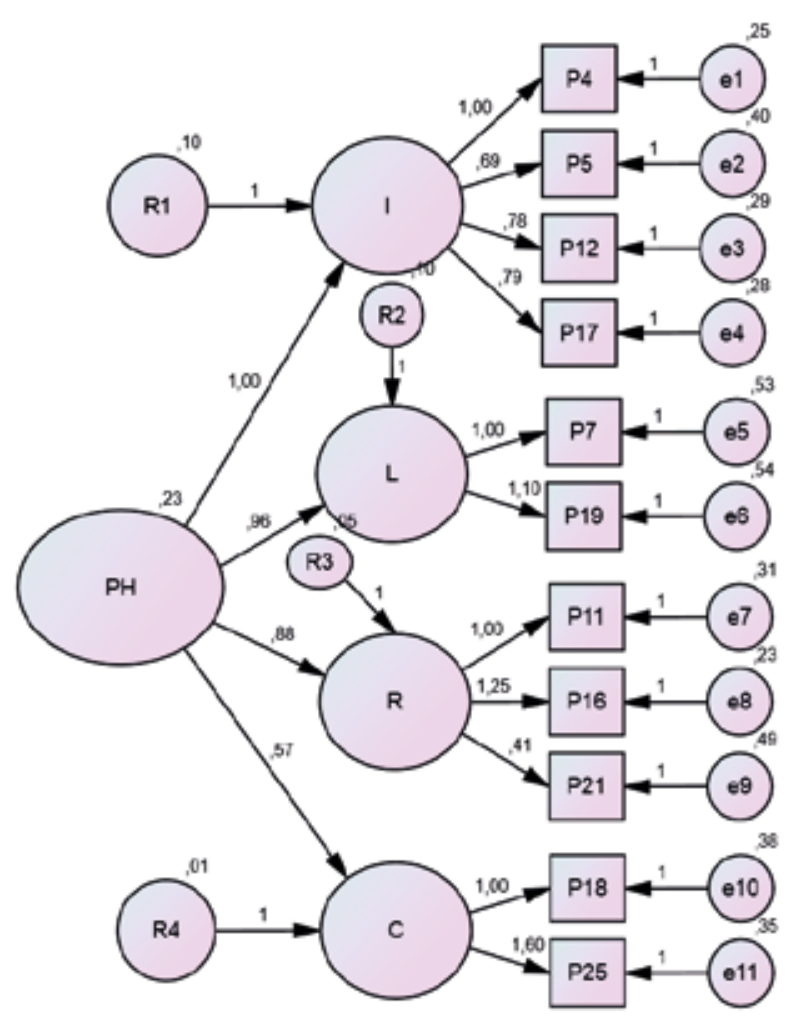

Figura 3. Estructura interna del modelo de cuatro dimensiones de la variable potencial humano. 
El análisis de consistencia interna, se realizó con el Alfa de Cronbach, el cual se calculó mediante el método de la varianza de los ítems; en la tabla 7, se observa las tres sub variables, con sus dimensiones y el puntaje específico para cada ítem si este fuera retirado, que en el caso de la variable cultura de la organización es mayor a 0,70; excepto del ítem 20 que resultó menor y la correlación elemento-total corregida fue superior a 0,30 con excepción del ítem 8. Respecto a la variable Diseño organizacional el puntaje específico para cada ítem si este fuera retirado resultó menor a 0,70 y la correlación elemento-total corregida fue superior a 0,30 excepto el ítem 10 y 34 . Respecto a la variable Potencial humano el puntaje específico para cada ítem si este fuera retirado resultó mayor a 0,78 y la correlación elemento-total corregida fue superior a 0,30 excepto el ítem 21. La consistencia interna global fue adecuada (Alfa=0,884), la sub variable Cultura de la organización mostró un coeficiente Alfa de 0,750; Diseño organizacional 0,671 (no aceptable) y Potencial humano 0,816 .

Tabla 7. Consistencia interna mediante coeficiente Alfa de Cronbach.

\begin{tabular}{|c|c|c|c|c|}
\hline Sub variables & Dimensiones & Ítems & $\begin{array}{c}\text { Correlación elemento -total } \\
\text { corregida }\end{array}$ & $\begin{array}{c}\text { Alfa si el ítems se } \\
\text { elimina }\end{array}$ \\
\hline \multirow{9}{*}{$\begin{array}{l}\text { Cultura de la } \\
\text { Organización }\end{array}$} & \multirow{3}{*}{ Motivación } & 1 & 0,443 & 0,724 \\
\hline & & 8 & 0,204 & 0,77 \\
\hline & & 33 & 0,491 & 0,715 \\
\hline & \multirow{3}{*}{ Identidad } & 20 & 0,654 & 0,68 \\
\hline & & 23 & 0,442 & 0,725 \\
\hline & & 31 & 0,365 & 0,738 \\
\hline & Conflicto & 24 & 0,44 & 0,725 \\
\hline & \multirow[t]{2}{*}{ Cooperación } & 26 & 0,554 & 0,704 \\
\hline & & & Alfa de Cronbach & 0,75 \\
\hline \multirow{10}{*}{$\begin{array}{l}\text { Diseño } \\
\text { Organizacional }\end{array}$} & Toma & 3 & 0,503 & 0,607 \\
\hline & Decisiones: & 14 & 0,312 & 0,652 \\
\hline & \multirow{2}{*}{ Remuneración } & 6 & 0,316 & 0,651 \\
\hline & & 27 & 0,353 & 0,643 \\
\hline & \multirow{2}{*}{ Estructura } & 10 & 0,109 & 0,684 \\
\hline & & 13 & 0,315 & 0,651 \\
\hline & \multirow{4}{*}{$\begin{array}{l}\text { Comunicación } \\
\text { Organizacional }\end{array}$} & 29 & 0,512 & 0,609 \\
\hline & & 30 & 0,455 & 0,617 \\
\hline & & 34 & 0,206 & 0,67 \\
\hline & & & Alfa de Cronbach & 0,671 \\
\hline \multirow{13}{*}{$\begin{array}{l}\text { Potencial } \\
\text { Humano }\end{array}$} & \multirow{4}{*}{ Innovación } & 4 & 0,618 & 0,788 \\
\hline & & 5 & 0,341 & 0,814 \\
\hline & & 12 & 0,573 & 0,793 \\
\hline & & 17 & 0,541 & 0,796 \\
\hline & \multirow{2}{*}{ Liderazgo } & 7 & 0,457 & 0,804 \\
\hline & & 19 & 0,533 & 0,796 \\
\hline & \multirow{3}{*}{ Recompensa } & 11 & 0,541 & 0,796 \\
\hline & & 16 & 0,627 & 0,787 \\
\hline & & 21 & 0,192 & 0,827 \\
\hline & \multirow{4}{*}{ Confort } & 18 & 0,366 & 0,811 \\
\hline & & 25 & 0,514 & 0,798 \\
\hline & & & Alfa de Cronbach & 0,816 \\
\hline & & & Alfa de Cronbach global & 0,884 \\
\hline
\end{tabular}




\section{DISCUSIÒN}

El presente trabajo de investigación, analiza las propiedades psicométricas del instrumento tipo escala para medir el clima organizacional del personal del Ministerio de Salud del Perú, en una muestra de trabajadores de la Dirección Regional de Salud Ica. La evaluación de las propiedades métricas presento ciertas debilidades en las la validez de constructo y fiabilidad, que discutimos a continuación.

El análisis de la viabilidad de los ítems, mostró índice de discriminación aceptables $(0,41$ a 0,89); dado que, al tratarse de una escala de tipo Likert, se acepta un valor mínimo de 0,20 para poder afirmar que los ítems discriminan (18); en consecuencia estos resultados significan que todos los reactivos discriminan adecuadamente.

Por otro lado, el análisis de matriz de correlación revelo valores de correlación ítems-total, desde 0,28 a 0,77; valores que indican una magnitud de correlación baja a moderada en la mayoría de los ítems del instrumento.

Las pruebas psicométricas utilizadas para justificar el análisis factorial fueron el test de esfericidad de Bartlett (que evalúa si la matriz de correlaciones es una matriz de identidad) y la medida de adecuación muestral KMO (que compara los coeficientes de correlación observados con los coeficientes de correlación parcial). En el presente estudio, el test de esfericidad de Barlett, tuvo un p-valor significativo, no obstante la medida de adecuación muestral KMO para la variable cultura de la organización fue 0,693 $(<0,70)$; diseño organizacional $0,589(<0,70)$; y potencial humano $0,811(>0,70)$. . Sin embargo, se procedió al realizar el AFC del instrumento, confirmando el supuesto teórico según el cual se basó el diseño del instrumento (tres dimensiones) para la variable cultura de la organización, el AFC para las variables diseño de la organización y potencial humano planteó tres dimensiones y no cuatro como se presenta en el instrumento original. De igual modo sugirió la reubicación de las dimensiones debido a su carga factorial y detecto que algunos reactivos no pertenecen a la dimensión asignada en el instrumento original. Para la variable general el test de esfericidad de Barlett, tuvo un p-valor significativo $(0,000)$ y la medida de adecuación muestral $\mathrm{KMO}=0,715$ y el $\mathrm{AFC}$, sugirió siete dimensiones cuando se excluye

los ítems de la escala de sinceridad y 11 dimensiones cuando se incluye los reactivos de la referida escala, pero las dimensiones y preguntas no se corresponden a la ubicación asignada en el instrumento. En consecuencia, nos encontramos con una estructura factorial poco consistente; sin embargo, con ítems que podrían medir la variable estudiada; que además cuenta con suficiente evidencia científica, que apoye al clima organizacional, como constructo claramente definido.

No obstante las medidas de calidad de ajuste de los modelos factoriales revelaron un $\mathrm{p}$-valor $<0,05$ (no apropiado) para la variable cultura de la organización y $>0,05$ (apropiado) para la variable diseño organizacional y potencial humano; las medidas de ajustes incremental mostraron valores no apropiados $(<0,90)$; así como, las medidas de ajuste de la parsimonia se situaron por debajo del valor esperado. Esto significa que los modelos factoriales no se ajustan de manera satisfactoria a los datos. Situación que podría ocasionar polémica sobre la estructura factorial del instrumento.

La consistencia interna del instrumento, obtuvo un coeficiente de Alfa de Cronbach de 0,88. A ese respecto Kline(21), establece que para pruebas de uso clínico lo recomendable es un índice de confiabilidad de 0,85 en adelante y para investigación de 0.70 hacia arriba. Por tanto, se trata de un instrumento fiable que realizará mediciones estables y consistentes. El rango para las tres variables osciló entre 0,67 a 0,81 ; no obstante la variable diseño organizacional fue la única variable con $a<0,70$. En este caso es necesario tomar en cuenta que dicha variable consta de 09 ítems y se conoce que entre más cantidad de ítems presente un instrumento, el a será mayor, debido a que tanto la extensión del cuestionario como el tamaño de la muestra influyen positivamente en el coeficiente (22). Sin embargo, también se ha considerado que un coeficiente muy alto puede indicar mayor redundancia, es decir, ítems que indagan por las mismas características de manera diferente.

En cuanto a las limitaciones del trabajo, podemos discutir si el tamaño de la muestra es suficiente para definir la estructura factorial del instrumento y si los trabajadores de la Dirección Regional de Salud Ica, son válidamente representativos del Ministerio de Salud del Perú. Esto creemos debe ser evaluado en próximos estudios que consideren un tamaño de muestra mayor y un muestreo estratificado y/o por conglomerados que incluya a toda la población de trabajadores del Ministerio de Salud del Perú. No obstante, el presente estudio analiza las propiedades psicométricas del instrumento, en un contexto espacial que ofrece un nivel de representatividad local y/o regional y emplea una técnica adecuada para el proceso de recolección de datos, disminuyendo la posibilidad de sesgo de información.

Los resultados obtenidos demuestran que la validez de constructo del instrumento presenta debilidades esto fue evidenciado al observar la correlación de los ítems y factores confirmatorios; sin embargo, la consistencia interna global mostró confiablidad aceptable, en consecuencia el instrumento puede servir de manera fiable para valorar el clima organizacional; por lo tanto, se recomienda su uso en futuras investigaciones. 


\section{REFERENCIAS BIBLIOGRÁFICAS}

1. Cardona DR, Zambrano R. Revisión de instrumentos de evaluación de clima organizacional. Estudios Gerenciales. 2014;30(131):184-9.

doi: https://www.doi.org/10.1016/j.estger.2014.04.007

2. Santa Eulalia J, Sampedro B. Clima organizacional en instituciones de atención primaria de salud. Revista Médica Electrónica. 2012 [citado 24 marzo 2020];34(5):606-19. Disponible en: https://www.goo.gl/Y7G6d8

3. Silva R, de la Torre J, López A, Bastos S. El clima organizacional en el diseño del Balanced Scorecard: evaluación psicométrica de un instrumento de medida. Revista Contabilidade Vista \& Revista. 2011;22(1):107-41. Disponible en: https://www.redalyc.org/pdf/1970/197017517005.pdf

4. Carmona V, Jaramillo E. Estudio del clima organizacional en la E.S.E. Hospital San Jorge de Pereira [tesis para obtención de grado]. Pereira, Colombia: Universidad Tecnológica de Pereira; 2010.

5. Pelaes O. Relación entre el clima organizacional y la satisfacción del cliente en una empresa de servicios telefónicos. Universidad Nacional Mayor de San Marcos. Programa Cybertesis PERÚ, [Internet] 2010 [citado 24 marzo 2020]. Disponible en: https://www.goo.gl/MZ5sxZ

6. Cújar $A$, Ramos $C$, Hernández $H$, López J. Cultura organizacional: evolución en la medición. Estudios Gerenciales. 2014;29(128):350-5. Disponible en: https://www.redalyc.org/articulo.oa?id=21229786009

7. Likert R. Una técnica para medir actitudes. Nueva York: Trillas; 1976.

8. Halpin A, Crofts D. The organizational climate of schools. International Review of Education. 1963;22(4):441-63. Disponible https://www.link.springer.com/article/10.1007/BF00598815

9. Litwin G, Stringer R. Motivation and organizational climate. Boston: Harvard University Press; 1968.

10. Pérez de Maldonado I, Maldonado M, Bustamante S. Clima organizacional y gerencia: inductores del cambio organizacional. Investigación y Postgrado [internet]. 2006 [citado 24 marzo 2020];21(2):231-48. Disponible en: https://www.goo.gl/TdWcV3

11. Segredo Pérez AM. Clima organizacional en la gestión del cambio para el desarrollo de la organización. Rev Cubana Salud Pública [Internet]. 2013 [citado 24

marzo 2020];39(2):385-93. Disponible en: https://www.goo.gl/onArbA

12. Ministerio de salud. Metodología para el estudio del clima organizacional, versión 02. Lima: MINSA; 2011. [citado 02 de febrero de 2020]. Disponible en: http://bvs.minsa.gob.pe/local/MINSA/2112.pdf

13. Calviño M. Cambiando la mentalidad. Empezando por los jefes. La Habana: Editorial Academia; 2014.

14. Dimensiones para evaluar el clima. c2000-2006 [citado 26 marzo 2020]. Disponible en: http://www.haygroup.com.ve/

15. Variables para estudiar el Clima Organizacional. Gestar consultoría. [citado 26 marzo 2020]. Disponible en: www.gestar.cl/cons

16. Serrate Alfonso A, Portuondo Vélez AL, Sánchez Puigbert N. Suárez Ojeda R. Evaluación de la cultura organizacional y su incidencia en la efectividad grupal. Rev Ingeniería Industrial. 2014 [citado 2 Abr 2015];35(1):2-12. Disponible en:

http://rii.cujae.edu.cu/index.php/revistaind/article/view/620/519
17. Segredo Pérez AM. Propuesta de instrumento para el estudio del clima organizacional. Rev Correo Científico Médico de Holguín. 2013 [citado 10 Mar 2015];17(3). Disponible http://www.revcocmed.sld.cu/index.php/cocmed/article/vi ew/328/403

18. Barbero M. Psicometría II. Métodos de elaboración de escalas. Madrid: Uned; 1993.

19. Pett M, Lackey N, Sullivan J. Making sense of factor analysis: the use of factor analysis for instrument development in health care research. California: Sage Publication; 2003. Acceso: 26/02/2020. Disponible en: https://dx.doi.org/10.4135/9781412984898

20. Campo-Arias A, Oviedo H. Propiedades psicométricas de una escala: La consistencia interna. Revista de Salud Pública. [Internet]. 2008; 10(5), 831-839. Acceso: 26/02/2020. Disponible https://www.redalyc.org/pdf/422/42210515.pdf

21. Kline P. The Handbook of psychometric testing. New York: Routledge. 2000.

22. Streiner DL, Norman GR. Selecting the Items. En: Health Measurement Scales. A Practical Guide to Their Developmental and Use. New York: Oxford University Press; 2008. p. $77-102$. 